Methods: Consecutive patients undergoing a LSG for clinically suspected pSS were included in this study from January 2018 to October 2020. UHFUS of LSG was performed by using VEVO MD, equipped with a $70 \mathrm{MHz}$ probe, scanning first the central compartment of the inferior lip, and then both peripheral compartments. Parenchymal homogeneity was graded on a scale of 0 (normal) to 3 (evident). UHFUS imaging was used to locate the LSG for the US-guided biopsy. For each of the LSG specimens the area of the glandular tissue, the presence and number of foci and the focus score (FS) were assessed. Immunostaining for CD21 was performed to evaluate the presence of follicular dendritic cells (FDC) in foci.

Results: We included a total of 171 patients with suspected pSS: out of them, 83 (48.5\%) received a diagnosis of pSS (ACR 2016 criteria) and $88(51.5 \%)$ were diagnosed as no-SS sicca controls. Out of the 171 LSG biopsies, 73/171 (42.7\%) were characterized by a nonspecific chronic sialadenitis (NSCS) whereas a focal lymphocytic sialadenitis (FLS) was described in the remaining 98/171 (57.3\%). UHFUS-LSG scores were differently distributed in pSS patients with respect to no-SS sicca controls: a score 0 was detected in 16/88 no-SS sicca controls and in none of the 83 pSS patients, whereas a score 3 was detected in 12/83 pSS and in none of no-SS sicca controls. $(p<0.001)$. The higher was the UHFUS grading, the higher was the number of foci (from a mean (S.D) $=0.38(0.72)$ in UHFUS-score 0 to 4.58 (3.11) in UHFUS-score $3, p<0.001$ ) and the focus score (from 0.17 (0.32) in UHFUS-score 0 to $1.88(0.90)$ in UHFUS-score $3, p<0.001$ ) of the samples. Overall, out of the 16 no-SS controls with LSG-UHFUS score 0 , only 4/16 showed histological features of focal lymphocytic infiltrate; however, the mean FS of those 4/16 samples was 0.17 (0.32). In fact, considering a $F S \geq 1$, negative UHFUS-LSG was highly predictive of a negative histology (100 \%). By contrast, out of the 12 pSS patients with the highest score at LSG-UHFUS (score 3), 10/12 presented FDC networks in their biopsies, with a positive predictive value of $83 \%$ of the severity of the infiltrate.

Conclusion: UHFUS of LSG appeared feasible and sensitive in pSS; due to its striking negative predictive value this novel tool can help to identify negative patients on subsequent lip biopsy, thus avoiding invasive procedures in selected cases. Besides its diagnostic role, considering the association with the presence of FDC networks, UHFUS-LGS may also have a role in patients prognostic stratification.

Disclosure of Interests: None declared

DOI: 10.1136/annrheumdis-2021-eular.3484

\section{POS0779 MYOCARDIAL INFARCTIONS, SUBTYPES, LOCATIONS AND CORONARY ATHEROSCLEROSIS IN SLE - A COMPARATIVE STUDY WITH MATCHED CONTROLS}

I. Samuelsson ${ }^{1}$, I. Parodis ${ }^{1,2}$, I. Gunnarsson ${ }^{1,2}$, A. Zickert ${ }^{1,2}$, C. HofmanBang $^{3,4}, \mathrm{H}$. Wallén ${ }^{3,4}$, E. Svenungsson ${ }^{1,2}$. ${ }^{1}$ Karolinska Institutet, Division of Rheumatology, Department of Medicine Solna, Stockholm, Sweden; ${ }^{2}$ Karolinska University Hospital, Unit of Rheumatology, Stockholm, Sweden; ${ }^{3}$ Karolinska Institutet, Department of Clinical Sciences, Danderyd Hospital, Stockholm, Sweden; ${ }^{4}$ Danderyd Hospital, Unit of Cardiology, Stockholm, Sweden

Background: Myocardial infarction (MI) is estimated to be 2- to 3-fold elevated in systemic lupus erythematosus (SLE) as compared to gender- and age-matched controls (1-2). Even though some risk factors have been purposed, mechanisms of increased $\mathrm{MI}$ incidence remains to be determined.

Objectives: To explore underlying mechanisms, we compared $\mathrm{Ml}$ characteristics and risk factors between SLE patients with MI (MI-SLE), MI patients without SLE (MI-nonSLE) and SLE patients without MI (nonMI-SLE).

Methods: We performed retrospective medical file review including angiography and echocardiography reports in $34 \mathrm{MI}$-SLE patients, $34 \mathrm{MI}$-nonSLE patients and 34 nonMI-SLE patients - all individually matched for gender and age in a ratio of $1: 1: 1$

Results: Median age was 61 years and $88 \%$ were females. MI-SLE patients had more coronary arteries involved (table $1 ; p=0.038$ ), and $\geq 50 \%$ coronary atherosclerosis/occlusion at $\mathrm{Ml}$ was numerically more common compared to MI-nonSLE controls ( $88 \%$ versus $66 \% ; p=0.065$ ). The left anterior descending artery was most frequently involved in both $\mathrm{Ml}$ groups $(73 \%$ versus $59 \%$; $p=0.11)$. Decreased $(<50 \%)$ left ventricular ejection fraction occurred with similar frequency ( $45 \%$ versus $36 \% ; p=0.79$ ) in MI-SLE patients compared to MI-nonSLE patients. Cardiovascular disease (CVD) $(44 \%, 5.9 \%, 12 \% ; p<0.001)$ and coronary artery disease excluding $\mathrm{MI}(\mathrm{CAD}, 32 \%, 2.9 \%, 0 \% ; \mathrm{p}<0.001)$ preceded $\mathrm{Ml} /$ inclusion more commonly in MI-SLE than in MI-nonSLE and nonMI-SLE patients, respectively. MI-SLE patients differed from nonMI-SLE patients through lower plasma albumin levels (35 (29-37) versus 40 (37-42) $\mathrm{g} / \mathrm{L} ; \mathrm{p}=0.002)$ and longer disease duration (22 (14-32) versus 14 (6.3-24) years; $p=0.038$ ).

Conclusion: We demonstrate that non-procedural Mls in SLE are in $88 \%$ of cases associated with significant coronary atherosclerosis. Increased prevalence of $\mathrm{CAD}$ prior $\mathrm{MI}$ and higher number of coronary arteries involved at MI, suggest accelerated coronary atherosclerosis in SLE patients. This calls for attentive surveillance of CVD and early atheroprotective treatment in this patients group.

\section{REFERENCES:}

[1] Hak AE et al. Systemic lupus erythematosus and the risk of cardiovascular disease: Results from the nurses' health study. Arthritis and rheumatism 2009;61:1396-402

[2] Fischer LM et. Effect of rheumatoid arthritis or systemic lupus erythematosus on the risk of first-time acute myocardial infarction. The American journal of cardiology 2004;93:198-200.

Table 1. MI characteristics

\begin{tabular}{|c|c|c|c|c|c|}
\hline & MI-SLE & $\mathrm{N}_{\text {total }}$ & MI-nonSLE & $\mathrm{N}_{\text {total }}$ & P-value \\
\hline ECG findings & $23(72 \%)$ & 32 & $21(66 \%)$ & 32 & 1.0 \\
\hline \multicolumn{6}{|l|}{ NSTEMI } \\
\hline STEMI & $9(28 \%)$ & 32 & $11(34 \%)$ & 32 & \\
\hline \multicolumn{6}{|l|}{ Presence of atherosclerosis } \\
\hline $0-V D$ & $3(12 \%)$ & 26 & $10(35 \%)$ & 29 & \multirow[t]{2}{*}{0.065} \\
\hline MI-CAD ( $\geq 1-V D)$ & $23(88 \%)$ & 26 & $19(66 \%)$ & 29 & \\
\hline \multicolumn{6}{|l|}{ Number of involved arteries } \\
\hline $0-V D$ & $3(12 \%)$ & 26 & $10(35 \%)$ & 29 & \multirow[t]{3}{*}{0.038} \\
\hline 1-VD & $13(50 \%)$ & 26 & $9(31 \%)$ & 29 & \\
\hline$\geq 2-V D$ & $10(39 \%)$ & 26 & $10(35 \%)$ & 29 & \\
\hline Involvement of specific arteries & $3(12 \%)$ & 26 & $0(0 \%)$ & 29 & 0.50 \\
\hline \multicolumn{6}{|l|}{ LMCA } \\
\hline LAD & 19 (73\%) & 26 & 17 (59\%) & 29 & 0.11 \\
\hline RCA & $7(27 \%)$ & 26 & $9(31 \%)$ & 29 & 0.75 \\
\hline$C x$ & $6(23 \%)$ & 26 & $6(21 \%)$ & 29 & 1.0 \\
\hline \multicolumn{6}{|l|}{ Left ventricular ejection fraction } \\
\hline$<50 \%$ & $13(45 \%)$ & 29 & $12(36 \%)$ & 33 & \multirow[t]{2}{*}{0.79} \\
\hline$\geq 50 \%$ & $16(55 \%)$ & 29 & $21(64 \%)$ & 33 & \\
\hline
\end{tabular}

$0-V D=0$-Vessel disease. $1-V D=1$-Vessel disease. $2-V D=2$-Vessel disease. $C x=$ Circumflex artery. $L A D=$ Left anterior descending artery. $L M C A=$ Left main coronary artery. $M I-C A D=M I$ with coronary artery disease. NSTEMI $=$ Non-ST-elevation MI. RCA $=$ Right coronary artery. STEMI = ST-elevation MI.

Disclosure of Interests: Isak Samuelsson: None declared, loannis Parodis Grant/research support from: The author declare that he has no conflict of interest related to this work, Iva Gunnarsson Grant/research support from: The author declare that she has no conflict of interest related to this work, Agneta Zickert: None declared, Claes Hofman-Bang: None declared, Håkan Wallén Grant/ research support from: The author declare that he has no conflict of interest related to this work, Elisabet Svenungsson Grant/research support from: The author declare that she has no conflict of interest related to this work DOI: 10.1136/annrheumdis-2021-eular.3547

\section{POS0780 SKIN LESIONS IN PATIENTS WITH SYSTEMIC LUPUS ERYTHEMATOSUS AND ANTIPHOSPHOLIPID SYNDROME}

A. Shumilova ${ }^{1}$, F. Cheldieva ${ }^{2,3}$, T. Reshetnyak ${ }^{2,3}$, A. Lila ${ }^{3,4} .{ }^{1}$ V.A. Nasonova Research Institute of Rheumatology, 4th Department of Rheumatology, Moscow Russian Federation; ${ }^{2}$ V.A. Nasonova Research Institute of Rheumatology, Vascular Rheumatology Department, Moscow, Russian Federation; ${ }^{3}$ Russian Medical Academy of Continuing Professional Education, Department of Rheumatology, Moscow, Russian Federation; ${ }^{4}$ V.A. Nasonova Research Institute of Rheumatology, Head of the Institute, Moscow, Russian Federation

Background: In more than $40 \%$ of cases, systemic lupus erythematosus (SLE) is associated with the presence of highly positive antiphospholipid antibodies with $50-70 \%$ of patients developing antiphospholipid syndrome (APS) over the next 10 years of the disease. Both diseases have similar and different clinical manifestations of skin lesions. The variety of skin lesions in SLE and APS requires a differential diagnosis and can make it difficult to diagnose a systemic autoimmune disease in a timely manner.

Objectives: To study the frequency of skin manifestations in SLE and APS, depending on the positivity of aPL.

Methods: The study included 116 patients with SLE (104 women and 12 men), mean age $37.9 \pm 12.9$, disease duration 8.5 [1.15-13.0]; 40 patients with APS (33 women and 7 men), mean age 36.2 \pm 9.39 . All patients were evaluated for skin lesions, and patients with APS were determined by $\mathrm{lgG} / \mathrm{lgM}-\mathrm{aCL}$ and $\mathrm{IgG} / \mathrm{lgM}$ aß2HP1 by enzyme immunoassay (ELISA), 19 of them were determined by $\lg$ AaCL, IgA-aß2HP1 and IgG-aß2HP1-D1 chemiluminescence analysis (CMA) Results: Acute skin lesions in past history were noted in $58(50 \%)$ patients, chronic lesions -I n 21 (18.1\%) patients; photosensivity and alopecia were indicated in $46(39.6 \%)$ patients, mucosal lesions were noted in $36(31 \%)$ of 116 patients, which corresponds to the literature data on the frequency of skin lesions and its appendages in SLE. At the time of inclusion in the study, skin lesions were detected in 20 patients. Score according to the CLASI index in patients with skin lesions: activity index=1.55 [0-22]; damage index=1.81 [0-36].

Skin lesions are the second most common signs of SLE onset (debut in 26 $(22.4 \%)$ patients), second only to arthritis (38 $(32.5 \%)$ patients), while the 